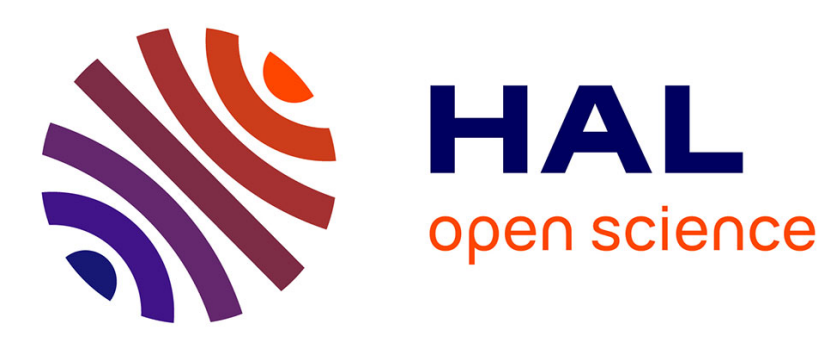

\title{
Efforts de production de parole chez les personnes qui bégaient
}

Maëva Garnier, Anaïs Dafonseca, Christophe Savariaux, Thibault Cattelain

\section{To cite this version:}

Maëva Garnier, Anaïs Dafonseca, Christophe Savariaux, Thibault Cattelain. Efforts de production de parole chez les personnes qui bégaient. JEP 2018 - 32e Journées d'Etudes sur la Parole, Jun 2018, Aix-en-Provence, France. hal-01867444

\section{HAL Id: hal-01867444 https://hal.science/hal-01867444}

Submitted on 4 Sep 2018

HAL is a multi-disciplinary open access archive for the deposit and dissemination of scientific research documents, whether they are published or not. The documents may come from teaching and research institutions in France or abroad, or from public or private research centers.
L'archive ouverte pluridisciplinaire HAL, est destinée au dépôt et à la diffusion de documents scientifiques de niveau recherche, publiés ou non, émanant des établissements d'enseignement et de recherche français ou étrangers, des laboratoires publics ou privés. 


\section{Efforts de production de parole chez les personnes qui bégaient}

Maëva Garnier, Anaïs DaFonseca, Christophe Savariaux, Thibault Cattelain, Univ. Grenoble Alpes, CNRS, Grenoble INP*, GIPSA-lab, 38000 Grenoble, France

* Institute of Engineering Univ. Grenoble Alpes maeva.garnier@gipsa-lab.grenoble-inp.fr

\section{RÉSUMÉ}

Cette étude vise à objective et quantifier les tensions orofaciales visibles et communément décrites chez des personnes qui bégaient (PQB), en comparant les efforts aréeodynamiques, laryngés et articulatoires lors de la production de consonnes occlusives entre des PQB et des personnes normofluentes (PNF). Dans cette étude préliminaire, 4 adultes qui bégaient et 4 adultes normofluents ont été enregistrés. Ils devaient produire des mots commençant par des consonnes occlusives labiales dans une tâche de lecture et dans une tâche semi-spontanée. Contrairement à nos attentes, les 4 PQB ont montré une force interlabiale (mesurée à partir d'un capteur de pression) et une pression intra-orale réduites par rapport aux 4 PNF. Aucune différence significative n'a été observée au niveau de ces paramètres entre les syllabes bégayées $\mathrm{v}$. perceptivement fluentes des PQB. Cependant, les différences de pression intra-orale observes entre les PQB et le PNF sont renforcées sur des syllabes complexes (commençant par un cluster consonantique) et pour la tâche semi-spontanée.

\section{ABSTRACT}

\section{Speech production efforts in people who stutter.}

This study aims at objecting and quantifying the visible oro-facial tensions that are commonly described in people who stutter (PWS), by comparing the aerodynamic, laryngeal and articulatory efforts of speech production between PWS and normo-fluent people (NFP). 4 adult PWS and 4 NFP were recorded, while producing words beginning with labial stop consonants in a reading task and a semi-spontaneous task. Contrary to our expectations, PWS demonstrated less intra-oral pressure and interlip force (measured with a force sensor) than NFP (with comparable syllable intensity, though). No significant difference in intra-oral pressure and interlip force was observed between the disfluent vs. perceptually fluent syllables produced by PWS. However, the differences in intra-oral pressure observed between PWS and NFP were greater on complex syllables (beginning with a consonant cluster) and for the semi-spontaneous task. The electroglottographic data is currently being analyzed and will be presented at the conference.

MOTS-CLES : Bégaiement, efforts de production, consonnes occlusives, pression intra-orale, force interlabiale.

KEYWORDS: Stuttering, production efforts, stop consonants, intra-oral pressure, interlip force.

\section{Introduction}

Le bégaiement est un trouble neuro-moteur se traduisant par des difficultés et des tensions lors de la production de parole, allant jusqu'à différents types de disfluences particulièrement audibles : des répétitions, des prolongations ou des blocages de sons. 
La question se pose encore de savoir si ce trouble est intermittent, n'existant qu'au moment des difluences perceptibles, provoqué par des facteurs extérieurs (stress, fatigue, charge cognitive, ...) (Caruso et al., 1994), ou bien si ce trouble est permanent, se traduisant à tout moment par des atypicités motrices, mais dont les conséquences acoustiques sont plus ou moins perceptibles et se répartissent sur un continuum de fluence (Peters et al. 2000).

La question se pose également de savoir quel(s) geste(s) sont altérés par ce trouble.

La plupart des études a porté sur la description de gestes laryngés atypiques (Montfrais-Pfauwadel 2005) et d'une activité musculaire laryngée excessive (Freeman \& Ushijima, 1978) au moment des disfluences. On dispose de moins d'information sur ce qui se passe au niveau articulatoire et respiratoire au moment des bégayages. Il a toutefois été montré que les disfluences perceptibles étaient généralement précédées de mouvements de la mâchoire (d'ouverture ou de fermeture) dont la vitesse était particulièrement élevée (Hutchinson and Watkin, 1976), d'anomalies au niveau des mouvements des lèvres et de la langue (Didirkova, 2016), associés à une augmentation plus rapide que la moyenne de la pression intra-orale pendant une occlusion (Hutchinson and Navarre, 1977). Par ailleurs, il semblerait que les gestes de production de parole des personnes qui bégaient continuent de montrer des particularités, même en dehors des instants de disfluences audibles. Ainsi, différents auteurs ont montré chez les PQB une hyper-excitabilité musculaire latente au niveau laryngé (Freeman \& Ushijima, 1978) et des difficultés à coordonner rapidement leurs mouvements laryngés et respiratoires pour initier ou terminer la vibration des plis vocaux (Adams 1974). Dans leur parole en apparence fluente, les PQB montrent également un débit oral expiré et une pression intra-orale significativement plus faibles que les PNF(Hutchinson et Navarre, 1977) et des défaillances du contrôle de la pression sous-glottique (Peters \& Boves, 1988). Des résultats beaucoup plus variables, voire contradictoires, sont reportés au niveau articulatoire : Van Lieshout et al. (1993) retrouvent, comme au niveau laryngé, des activités musculaires plus élevées au niveau de la lèvre inférieure des PQB, durant ou précédant la parole. Mais d'autres études n'observent aucune différence significative d'activité musculaire orofaciale entre des PQB et des PNF. Certains auteurs (Smith, 1989; Denny and Smith, 1992 ; De Felicio et al. 2007) observent même, au contraire, une activité musculaire inférieure à la moyenne au niveau de la lèvre supérieure. Une réduction vocalique est classiquement observée chez les personnes qui bégaient (Hirsch, 2007; Blomgren et al., 1998; Klich and May, 1982), supportant l'idée de gestes articulatoires moins amples. Pour autant, seul Zimmermann (1980) et McClean \& Runyan (2000) observent effectivement des mouvements moins amples et moins rapides de la lèvre inférieure et de la mâchoire. D'autres études ne trouvent aucune différence significative d'amplitude, de vitesse et/ou de durée des mouvements des lèvres ou de la mâchoire dans la parole perceptivement fluente de PQB, comparées à des PNF (McClean et al., 1990; Loucks et al., 2007). Enfin d'autres études encore rapportent de plus grandes amplitudes, de plus grands pics de vitesse, et de plus grandes durées des mouvements de la lèvre supérieure et de la langue chez les personnes qui bégaient (Namasivayam \& van Lieshout, 2008; Zmarich, 1994; 2001 ; Max et al., 2003; McClean and Runyan, 2000). A notre connaissance, aucune information n'a été apportée quant à la force des mouvements articulatoires d'occlusion.

Notre objectif est ici de décrire et comprendre ce que se passe au niveau du geste de production de parole lorsque la personne bégaye mais aussi le reste du temps lorsque sa parole semble en apparence fluente. En nous intéressant aux niveaux respiratoire et articulatoire, nous nous proposons

- d'observer les manifestations d'effort et les indices de difficultés de coordination entre les différents niveaux observés

- d'objectiver les tensions et les difficultés de production ressenties par les personnes qui bégaient en mesurant quantitativement la force articulatoire interlabiale, la pression intra-orale et l'intensité acoustique de consonnes occlusives bilabiales $(/ \mathrm{p} /, / \mathrm{b} /, / \mathrm{m} /)$. 
- d'apporter des éléments de réponse quant à l'aspect local ou global du bégaiement et quant à sa nature permanente ou intermittente, en comparant les efforts respiratoires, laryngés et articulatoires entre des locuteurs adultes qui bégaient et des locuteurs sans troubles de la parole, et entre les productions fluentes et disfluentes de personnes qui bégaient.

Nous formulons et testons ici 4 hypothèses :

H1 : Les personnes qui bégayent produiraient des consonnes occlusives bilabiales avec plus d'effort articulatoire et respiratoire que les personnes sans trouble de la parole

$\mathrm{H} 2$ : Des différences significatives de production de la parole seraient particulièrement observées pendant les bégayages de personnes qui bégaient, comparés à la parole de personnes normofluentes, mais également de façon moindre dans les autres segments de parole perceptivement fluents des personnes qui bégaient

H3 : Ces différences significatives de production de la parole entre personnes normofluentes et personnes qui bégaient seraient amplifiées par la complexité phonologique.

$\mathrm{H} 4$ : Ces différences significatives de production de la parole entre personnes normofluentes et personnes qui bégaient seraient observées uniquement, ou davantage, en tâche semi-spontanée par rapport à une tâche de lecture.

\section{Matériel et méthodes}

Quatre adultes qui bégaient (PQB) (2 hommes, 2 femmes) et quatre adultes normofluents (PNF) (3 hommes, 1 femme), âgés de 20 à 46 ans, tous francophones, ont participé à cette étude préliminaire, réalisée à l'occasion du mémoire d'orthophonie d'Anaïs Da Fonseca (2016). Les quatre PQB présentaient un bégaiement développemental et avaient suivi des rééducations orthophoniques par le passé (de 3 à 5 ans selon les sujets, avec des méthodes variables dont ERASM). Plus aucun participant ne suivait de rééducation au moment de l'expérience.

Les participants ont été enregistrés dans deux conditions expérimentales : 1) une condition noninteractive de lecture de listes de mots le plus rapidement possible et 2) une condition de production semi-spontanée de ces mêmes mots : l'expérimentateur, assis en face du participant, retournait sur la table des séries de 3 cartes représentant les mots cibles. Le participant devait rapidement inventer une phrase à partir de ces 3 mots. Chaque série était répétée 5 fois dans un ordre pseudo-aléatoire afin de composer des phrases différentes pour chaque série.

Le tableau 1 récapitule les 30 mots cibles de l'expérience. 18 d'entre eux ont été sélectionnés pour être constitués de 3 syllabes (CVCVCV), avec $/ \mathrm{p} /, / \mathrm{b} /$ ou $/ \mathrm{m} /$ en consonne initiale ${ }^{1}$, suivie de la voyelle $/ \mathrm{a} /, / \mathrm{i} / \mathrm{ou} / \mathrm{o} /$, et de genre masculin de façon à ce que la consonne occlusive soit précédée de la voyelle /e/. Les 12 mots restants ont été sélectionnés sur le modèle, mais débutant par les clusters consonantiques $/ \mathrm{pR} /$ et $/ \mathrm{bR} /$ de façon à examiner l'effet de la complexité articulatoire.

Quatre signaux ont été acquis simultanément :

- La pression intra-orale a été enregistrée à l'aide d'un fin tube capillaire maintenu dans la cavité buccale, relié à un pneumotachographe (station d'acquisition de données physiologiques EVA).

- Le signal audio de parole a été enregistré à l'aide d'un microphone de pression (Bruël and Kjær 4944-A) placé à $30 \mathrm{~cm}$ des lèvres. Le niveau d'intensité acoustique était calibré à l'aide d'un amplificateur de mesure (Nexus, Bruel\&Kjaer) délivrant un signal interne de référence à $1 \mathrm{kHz}$.

- Le signal électroglottographique a été enregistré à l'aide d'un électroglottographe (EG2 Glottal Enterprise).

1 Les personnes qui bégaient rencontrent des difficultés particulières sur les consonnes occlusives bilabiales (Didirkova 2016) 
- La force de compression interlabiale a été mesurée à l'aide d'un capteur de pression à jauge de contrainte, collé sur la lèvre inférieure (Jeannin et al. 2008 ; Garnier et al. 2014).

- Ces trois derniers signaux ont été numérisés à $44100 \mathrm{~Hz}$ sur une même carte d'aquisition (Biopac) et post-synchronisés avec le signal de Pio acquis sur la station EVA.

\begin{tabular}{|l|l|l|l|l|l|}
\hline & $/ \mathbf{p} /$ & $/ \mathbf{p R} /$ & $/ \mathbf{b} /$ & $/ \mathbf{b R} /$ & $/ \mathbf{m} /$ \\
\hline \multirow{2}{*}{$/ \mathbf{a} /$} & $\underline{\text { Paradis }}$ & $\underline{\text { Praticien }}$ & $\underline{\text { Bananier }}$ & $\underline{\text { Brasero }}$ & $\underline{\text { Macaron }}$ \\
\cline { 2 - 6 } & $\underline{\text { Panama }}$ & $\underline{\text { Praliné }}$ & $\underline{\text { Baluchon }}$ & $\underline{\text { Braconnier }}$ & Maquillage \\
\hline \multirow{2}{*}{$/ \mathbf{i} /$} & $\underline{\text { Pissenlit }}$ & $\underline{\text { Prisonnier }}$ & $\underline{\text { Bikini }}$ & $\underline{\text { Bricoleur }}$ & Mirabelle \\
\cline { 2 - 6 } & $\underline{\text { Piranha }}$ & $\underline{\text { Professeur }}$ & $\underline{\text { Bijoutier }}$ & $\underline{\text { Britannique }}$ & Minibus \\
\hline \multirow{2}{*}{$/ \mathbf{o} /$} & $\underline{\text { Potager }}$ & $\underline{\text { Promoteur }}$ & $\underline{\text { Boléro }}$ & Brocoli & Mocassin \\
\cline { 2 - 6 } & $\underline{\text { Policier }}$ & $\underline{\text { Privation }}$ & $\underline{\text { Bolognaise }}$ & $\underline{\text { Brocanteur }}$ & Mobilier \\
\hline
\end{tabular}

TABLE 1 : Liste des 30 mots cibles visant à examiner la production de consonnes occlusives dans différents contextes vocaliques et dans des clusters consonantiques.

Les données ont été étiquetées manuellement sous Praat en repérant :

- le début et la fin des mots-cibles

- le début et la fin des syllabes initiales (CV ou CCV)

A l'aide de scripts développés sous Matlab, différents descripteurs ont ensuite été extraits des signaux enregistrés sur ces différents intervalles de temps :

- le maximum de pression intra-orale pendant la phase d'occlusion de la consonne (Piomax).

- le maximum de force interlabiale pendant la phase d'occlusion de la consonne (Fmax).

- l'intensité acoustique de la syllabe (Imax).

Les analyses statistiques ont été réalisées avec le logiciel $\mathrm{R}$ sur les syllabes fluentes des PQB et PNF. Pour chaque descripteur, nous avons modélisé les données à l'aide de modèles mixtes, incluant un effet aléatoire « Locuteur» et trois facteurs à effet fixe :

- Catégorie de sujet (2 niveaux : PQB vs. PNF). Les sujets n'étaient pas appariés.

- Tâche (2 niveaux : lecture vs. semi-spontanée)

- Consonne ou cluster de consonnes en début de mot ( 5 niveaux : /p/, /b/, /m/, /pr/, /br/)

Nous avions choisi 3 contextes vocaliques /a/, /i/ et /o/ non pas pour étudier l'influence du contexte vocalique mais plutôt pour que nos résultats ne soient pas spécifiques à une voyelle donnée. C'est pourquoi, en l'absence d'hypothèse sur l'effet du contexte vocalique, nous ne l'avons pas considéré dans les facteurs du modèle statistique.

Suivant la même procédure que celle développée avec des experts statisticiens, appliquée à l'analyse de plusieurs jeux de données précédents (Bourne et al. 2016), nous avons commencé par chercher à simplifier chaque modèle en excluant toute interaction non significative entre les facteurs à effet fixe. Pour cela, nous avons utilisé la fonction step sous R. Une fois le modèle simplifié, nous avons vérifié sa validité en examinant ses résidus. Nous avons ensuite réalisé des tests de modèle emboités (ou Likelihood Ratio Test) pour tester la significativité de chaque facteur ou de leurs interactions. Enfin, nous avons réalisé des tests posthoc pour examiner le contraste plus spécifique entre certaines conditions, en appliquant des corrections de Bonferroni pour les comparaisons multiples.

$\mathrm{Du}$ fait de l'effectif réduit des syllabes bégayées, la significativité des différences entre syllabes bégayées et perceptivement fluentes des PQB n'a pu être testée statistiquement. 


\section{Résultats}

La Figure 1 représente le taux de bégayages pour chacune des 4 personnes qui bégaient, en fonction de la syllabe initiale des mots et en fonction de la tâche de parole. En revanche, le niveau de bégayage est assez comparable entre les 4 individus de notre expérience, en moyenne de $2.72 \%$.

Contrairement à nos attentes, les bégayages ne sont pas significativement plus fréquents sur les syllabes phonologiquement plus complexes. Les bégayages les plus fréquents sont effectivement observés sur les mots-cibles commençant par $/ \mathrm{pR} /$, mais cette tendance ne s'étend pas aux motscibles commençant par $/ \mathrm{bR} /$. Conformément à nos attentes, les bégayages tendent à être plus fréquents sur la tâche semi-spontanée, comparée à celle de lecture des mots-cibles.

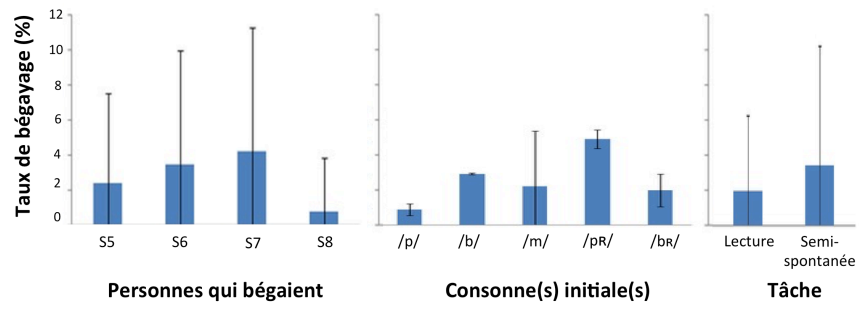

FIGURE 1 : Taux de bégayages (moyenne et écart-type) en fonction des participants, de la syllabe initiale et de la tâche.

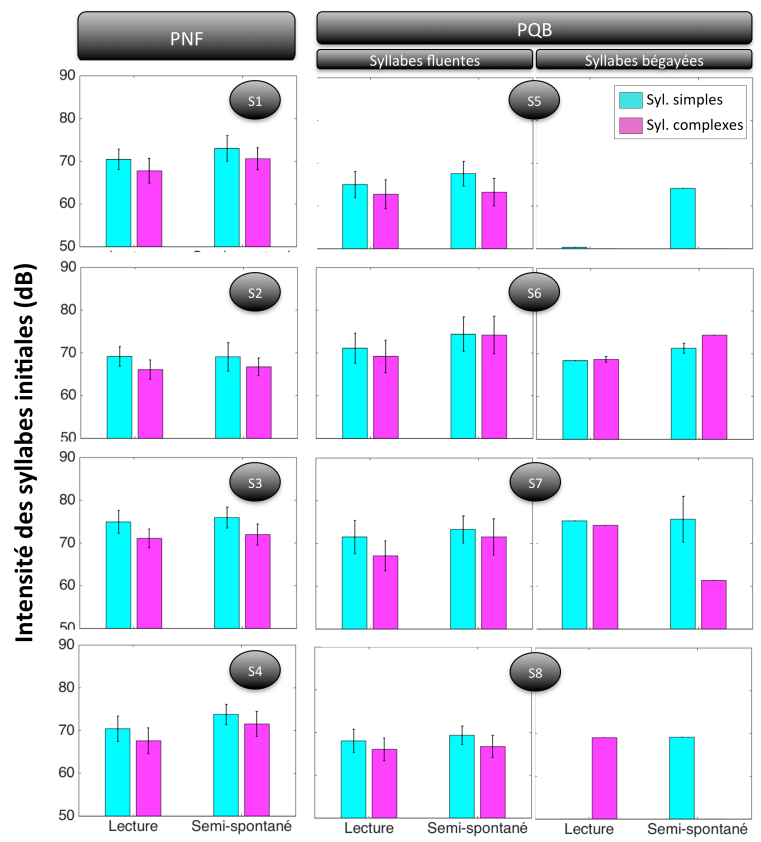

FigURE 2 : Intensité acoustique (moyennes et écart-types) des syllabes initiales chez les 4 personnes qui bégaient (PQB), pour les syllabes perceptivement fluentes ou bégayées et des 4 personnes normo-fluentes (PNF), en fonction de la tâche de l'expérience (lecture ou semi-spontanée) et de la complexité de la syllabe.

La Figure 2 synthétise les résultats obtenus quant à l'intensité acoustique des syllabes initiales. L'analyse statistique a montré que le modèle Intensité $\sim$ Tache*TypeSyllabe expliquait le mieux la 
variance de l'intensité vocale. Le facteur Groupe n'apparaît pas dans ce modèle simplifié, traduisant le fait que qu'il n'a pas d'effet significatif sur l'intensité des syllabes produites $(\mathrm{df}=1$, LRatio $=1.1, \mathrm{p}>0.2$ ). En d'autres termes, les 4 personnes qui bégaient de notre expérience parlent avec une intensité comparable aux 4 personnes normo-fluentes.

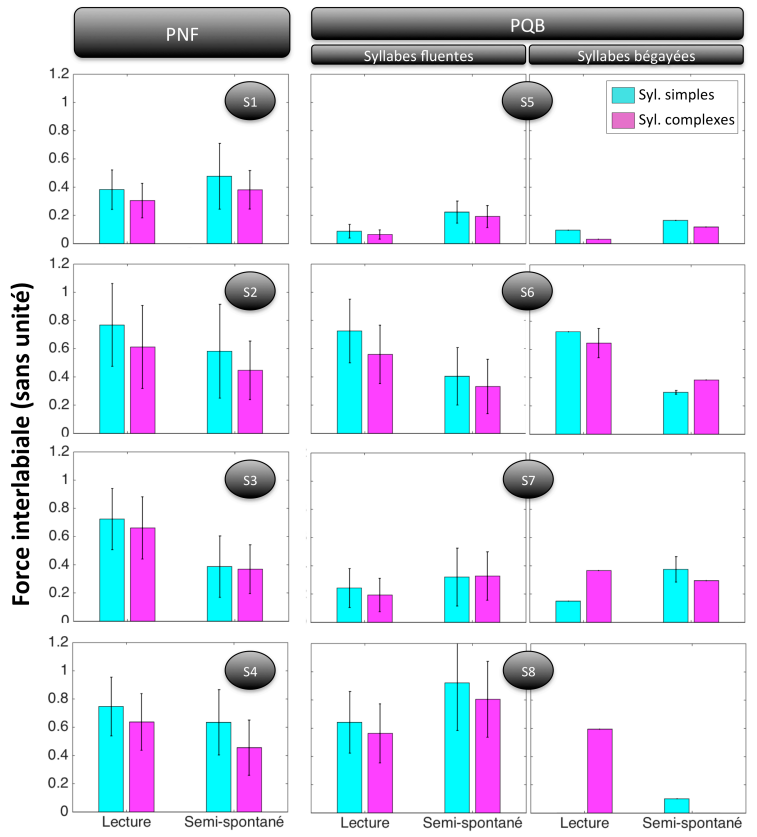

FIGURE 3: Force interlabiale (moyennes et écart-types) chez les 4 personnes qui bégaient (PQB), pour les syllabes perceptivement fluentes ou bégayées des 4 personnes normo-fluentes (PNF), en fonction de la tâche de l'expérience (lecture ou semi-spontanée) et de la complexité de la syllabe

La Figure 3 synthétise les résultats obtenus quant à la force articulatoire interlabiale mesurée sur les consonnes occlusives initiales des mots-cibles. L'analyse statistique a montré que le modèle Force $\sim$ Groupe*Tâche + Groupe*TypeSyllabe expliquait le mieux la variance de la force interlabiale.

Il existe un effet d'interaction significatif entre le Groupe et la Tâche $(\mathrm{df}=1$, Lratio=63.02, $\mathrm{p}<.0001^{* * *}$ ), traduisant le fait qu'en moyenne, les 4 personnes qui bégaient produisent des syllabes initiales avec une force articulatoire plus faible que les 4 personnes normofluentes en tâche de lecture $(-0.22$ (s.u), $\mathrm{p}=0.015, *)$, mais comparable lors de la tâche semi-spontanée $(-0.03$, $\mathrm{p}>0.9$ ).

Par ailleurs, il existe également un effet d'interaction significatif entre le Groupe et le Type de syllabe initiale $\left(\mathrm{df}=4\right.$, Lratio $\left.=21.28, \mathrm{p}<.0001^{* * *}\right)$, mais celui-ci ne provient pas $\mathrm{du}$ fait que, en moyenne, la différence de force interlabiale entre les 4 PQB et les 4 PNF soit particulièrement accentuée ou diminuée par la complexité phonologique $(\Delta=0.03, p>0.3)$. Individuellement, on observe que tous les $4 \mathrm{PNF}$ et 2 des PQB diminuent leur force articulatoire sur des syllabes complexes (commençant par $/ \mathrm{pR} /, / \mathrm{bR} /$ ), comparée à des syllabes simples (commençant par $/ \mathrm{p} /$, $/ \mathrm{b} /, / \mathrm{m} /$ ). Les deux autres PQB (S5, S7) ne varient pas significativement leur force interlabiale avec la complexité phonologique.

Enfin, la comparaison des syllabes bégayées et perceptivement fluentes produites par les 4 PQB ne montre pas de variation notable du niveau de force interlabiale (cf. Figure 3). 


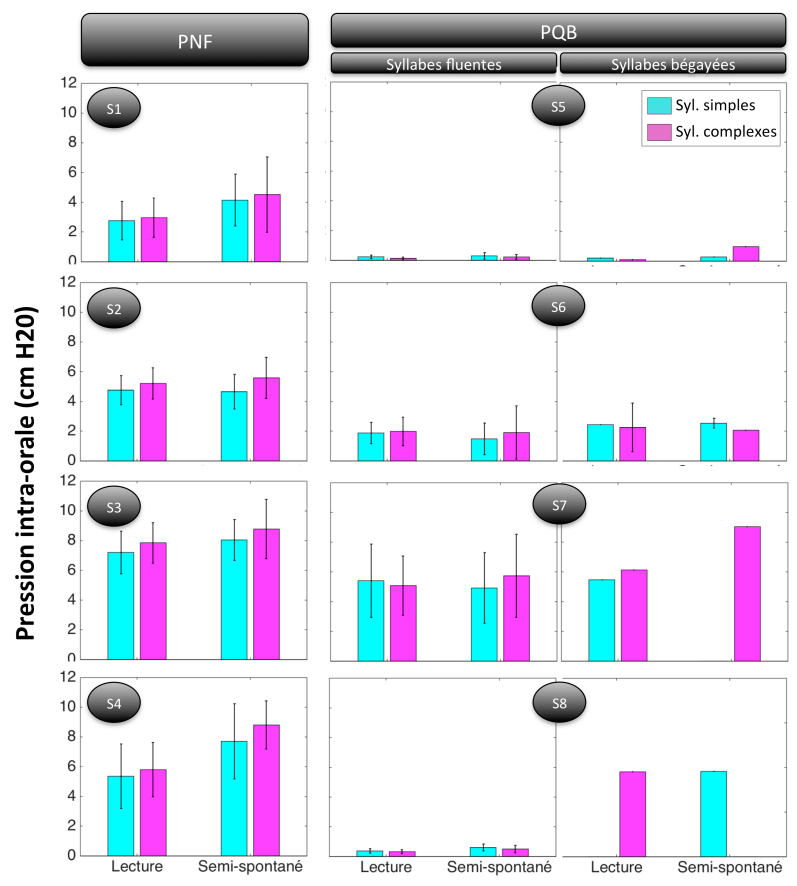

FIGURE 4: Pression intraorale chez les 4 personnes qui bégaient (PQB), pour les syllabes perceptivement fluentes ou bégayées des 4 personnes normo-fluentes (PNF), en fonction de la tâche de l'expérience (lecture ou semi-spontanée) et de la complexité de la syllabe

La Figure 4 synthétise les résultats obtenus quant à la pression intra-orale mesurée sur les consonnes occlusives initiales orales des mots-cibles $(/ \mathrm{p} /, / \mathrm{b} /)$ (la pression intra-orale est proche de zéro pour une occlusive nasale $/ \mathrm{m} /$, du fait que l'air continue de s'écouler par le nez).

L'analyse statistique a montré que le modèle global Pio $\sim$ Groupe*Tâche*TypeSyllabe expliquait le mieux la variance de la pression intra-orale.

Malgré, l'interaction significative observée entre les trois facteurs $(\mathrm{df}=3$, Lratio $=11.49, \mathrm{p}=0.009$ $(* *))$, une différence très claire se dégage entre les $4 \mathrm{PQB}$ et les 4 PNF de notre expérience, avec des niveaux de Pio significativement plus faibles chez PQB comparés aux PNF $(-3.9 \mathrm{~cm} \mathrm{H} 2 \mathrm{O}$ en moyenne, $\mathrm{p}=0.003 * *)$.

Nous remarquons que cet écart de Pio entre les PQB et PNF est significativement plus marqué en tâche semi-spontanée qu'en tâche de lecture. $(\Delta=+1.2 \mathrm{~cm} \mathrm{H} 20, \mathrm{p}<.001 * * *)$. En effet, on observe individuellement que les 4 locuteurs bègues, ainsi que le locuteur normo-fluent $\mathrm{S} 2$ ne varient pas significativement leur niveau de Pio entre les 2 tâches, tandis que les 3 autres locuteurs normofluents (S1, S3 et S4) augmentent leur Pio entre la tâche de lecture et la tâche de parole semi-spontanée (cf. Figure 4). 
On note également que l'écart de Pio entre les PQB et PNF est légèrement plus marqué pour les syllabes complexes que les syllabes simples. $(\Delta=+0.5 \mathrm{~cm} \mathrm{H} 20, \mathrm{p}=0.01 *)$. Individuellement, on observe en effet que tous les locuteurs normofluents augmentent leur Pio sur des syllabes complexes (commençant par $/ \mathrm{pR} /, / \mathrm{bR} /$ ), comparées à des syllabes simples (commençant $\mathrm{par} / \mathrm{p} /$, $/ \mathrm{b} /, / \mathrm{m} /$ ), tandis qu'aucun locuteur bègue ne montre de variation du niveau de Pio avec la complexité phonologique.

Enfin, la comparaison des syllabes bégayées et perceptivement fluentes produites par les 4 PQB ne montre toujours pas de variation notable du niveau de pression intra-orale pour 3 des locuteurs (S5, S6, S7). Seul le locuteur S8 montre une grande augmentation de pression intra-orale lors des bégayages (cf. Figure 4).

\section{Discussion et conclusion}

Contrairement à notre hypothèse, les $4 \mathrm{PQB}$ de cette étude ont montré moins de Pio et de force interlabiale que les PNF (malgré une intensité vocale comparable). Aucune différence significative de Pio et de force interlabiale n'a été observé entre les syllabes bégayées vs. perceptivement fluentes des PQB. Les différences de Pio observées entre nos 4 PQB et nos 4 PNF étaient plus importantes sur des syllabes complexes et pour une tâche semi-spontanée, ce qui n'était pas le cas pour la force inter-labiale.

Bien que contraires à nos attentes, les moindres forces aérodynamiques et articulatoires observées chez les PQB sont cohérentes avec d'autres observations reportées par de précédents études sur le bégaiement, en particulier une pression intra-orale (Pio) plus faible chez des PQB (Hutchinson \& Navarre, 1977), une amplitude et des vitesses réduites des gestes articulatoires (McClean \& Runyan, 2000; Zimmermann, 1980; Zmarich, 2001), une réduction vocalique...) (Blomgren, Robb, \& Chen, 1998; Hirsch, 2007; Klich \& May, 1982).

Il est possible que les niveaux plus faibles de force interlabiale et de pression intra-orale mesurés chez les 4 PQB de cette étude reflètent effectivement un certain niveau de décontraction, correspondant à la mise en œuvre de techniques d'aide à la fluence du type ERASM, à laquelle nos 4 PQB, comme la plupart des adultes

Bègues, sont formés à un moment ou un autre de leur parcours thérapeutique. Une autre explication serait que les tensions faciales visibles chez les personnes qui bégaient et leurs niveaux d'activité musculaire plus élevés ne soient pas nécessairement reliés à une amplification des gestes articulatoires ou respiratoires. Au contraire, certains auteurs ont pu observer une co-contraction de muscles antagonistes au niveau laryngé (Freeman \& Ushijima, 1978), pouvant expliquer, selon ces auteurs, la lenteur des mouvements d'adduction/abduction laryngée. Il est donc tout à fait envisageable qu'il en soit de même au niveau articulatoire et respiratoire, et que les tensions faciales visibles des personnes qui bégaient et leurs niveaux d'activité musculaire plus élevés témoignent en fait de crispations et de co-contraction de groupes de muscles antagonistes, ce qui aurait plutôt pour effet de réduire, voire de bloquer les mouvements, plutôt que d'amplifier leur force comme nous le pensions initialement.

\section{Remerciements}

Nous remercions les 8 participants à cette expérience. Cette recherche est financée par l'Agence Nationale de la Recherche (Projet StopNCo : Effort et coordination dans la production des consonnes occlusives ; ANR-14-CE30-0017; Maëva Garnier). 


\section{Réferences}

ADAMS, M. R. (1974). A physiologic and aerodynamic interpretation of fluent and stuttered speech. Journal of Fluency Disorders, 1(1), 35-47.

Bourne T., GARniER M. ET SAMSON A. (2016). Physiological and acoustic characteristics of the male music theatre voice. The Journal of the Acoustical Society of America, 140(1), 610-621

Blomgren, M., RobB, M., \& CHEN, Y. (1998). A note on vowel centralization in stuttering and nonstuttering individuals. Journal of Speech, Language, and Hearing Research, 41(5), 1042-1051.

Caruso, A. J., Chodzko-Zajko, W., Bidinger, D., \& Sommers, R. (1994). Adults Who Stutter: Responses to Cognitive Stress. Journal of Speech Language and Hearing Research, 37(4), 746.

DA FONSECA A. (2016). Effort articulatoire et respiratoire : étude de la parole bègue. Mémoire d'orthophonie, Université de Franche Comté.

DE Felício, C., Freitas, R., VitTi, M., \& Regalo, S. (2007). Comparison of upper and lower lip muscle activity between stutterers and fluent speakers. International Journal of Pediatric Otorhinolaryngology, 71(8), 1187-1192.

DenNY, M., \& SMITH, A. (1992). Gradations in a Pattern of Neuromuscular Activity Associated With Stuttering. Journal of Speech Language and Hearing Research, 35(6), 1216.

Didirkova, I. (2016) Parole, langues et disfluences : une étude linguistique et phonétique du bégaiement. Thèse de doctorat de l'Université Paul Valéry - Montpellier III.

Freeman, F., \& UshiJima, T. (1978). Laryngeal muscle activity during stuttering. Journal of Speech, Language, and Hearing Research, Vol. 21, 538-562.

GARNIER, M., BOUHAKE, S., ET JEANNIN, C. (2014) Efforts and coordination in the production of bilabial consonants. In 10th International Seminar on Speech Production. pp 138-141.

HIRSCH, F. (2007). Le bégaiement: perturbation de l'organisation temporelle de la parole et conséquences spectrales. Thèse de doctorat de l'Université de Strasbourg 2.

HutChinSON, J., \& NAVARRE, B. (1977). The effect of metronome pacing on selected aerodynamic patterns of stuttered speech: Some preliminary observations and interpretations. Journal of Fluency Disorders, 2(3), 189-204.

HUTCHINSON, J., \& WATKIN, K. (1976). Jaw mechanics during release of the stuttering moment : Some initial observations and interpretations. Journal of Communication Disorders, 9(4), 269- 279.

Jeannin, C., Perrier, P., Payan, Y., Grosgogeat, B., Dittmar, A., \& Géhin, C. (2009). PReSla: An original device to measure the mechanical interaction between tongue and teeth or palate during speech production. In Proceedings of International Seminar on Speech Production.

KLICH, R., \& MAY, G. (1982). Spectrographic study of vowels in stutterers' fluent speech. Journal of Speech, Language, and Hearing Research, 25(3), 364-370.

van Lieshout, P., Peters, H., Starkweather, C., \& Hulstijn, W. (1993). Physiological Differences Between Stutterers and Nonstutterers in Perceptually Fluent Speech: EMG Amplitude and Duration. Journal of Speech Language and Hearing Research, 36(1), 55.

LOUCKS, T. M. J., DE NiL, L. F., \& SASiSEKARAN, J. (2007). Jaw-phonatory coordination in chronic developmental stuttering. Journal of Communication Disorders, 40(3), 257-272.

MAX, L., CARUSO, A., \& GraCCO, V. (2003). Kinematic Analyses of Speech, Orofacial Nonspeech, and Finger Movements in Stuttering and Nonstuttering Adults. Journal of Speech Language and Hearing Research, 46(1), 215.

MCClean, M., Kroll, R., \& Loftus, N. (1990). Kinematic Analysis of Lip Closure in Stutterers' Fluent Speech. Journal of Speech Language and Hearing Research, 33(4), 755.

MCClEAN, M., \& RUNYAN, C. (2000). Variations in the relative speeds of orofacial structures with stuttering severity. Journal of Speech, Language, and Hearing Research, 43(6), 1524-1531.

McClean, M., GoldSmith, H., \& Cerf, A. (1984). Lower-Lip EMG and Displacement During Bilabial Disfluencies in Adult Stutterers. Journal of Speech Language and Hearing Research, 27(3), 342.

Monfrais-Pfauwadel, M.-C., Tromelin, O., Mougin, A.-L., \& OrmezZAno, Y. (2005). Utilisation des explorations multimédia synchrones dans l'objectivation des événements laryngés lors des bégayages. In Revue de laryngologie, d'otologie et de rhinologie (Vol. 126, p. 341-345).

NAMASIVAYAM, A. K., \& VAN Lieshout, P. (2008). Investigating speech motor practice and learning in people who stutter. Journal of Fluency Disorders, 33(1), 32-51.

Peters, H. \& BOVES, L. (1988). Coordination of aerodynamic and phonatory processes in fluent speech utterances of stutterers. Journal of Speech, Language, and Hearing Research, 31(3), 352-361.

Peters, H. M., Hulstijn, W., \& VAn Lieshout, P. H. (2000). Recent developments in speech motor research into stuttering. Folia Phoniatrica et Logopaedica, 52(1-3), 103-119.

SMith, A. (1989). Neural Drive to Muscles in Stuttering. Journal of Speech Language and Hearing Research, $32(2), 252$.

ZimmermanN, G. (1980). Articulatory Dynamics of Fluent Utterances of Stutterers and Nonstutterers. Journal of Speech Language and Hearing Research, 23(1), 95.

ZMARICH. (1994). Articulatory kinematics of lips and jaw in reiterant/pa/and/ba/sequences in Italian stutterers. Journal of Fluency Disorders.

ZMARICH, C. (2001). What Phonetics has to say about stuttering. Lecture held at the Socrates European Intensive Programme for Speech and Language Therapy, Diploma Universitario di Logopedia, Collegio Mazza, Padova, 22, 2000. 